\title{
Descripción del consumo de drogas lícitas e ilícitas por género a través de la metodología de pares
}

\author{
Jorge Rodríguez $\mathrm{T}^{\mathrm{la}}$, Enrique Hernández $\mathrm{A}^{\mathrm{lb}}$, \\ Ana María Fernández $2 c$. \\ A gender comparison of legal \\ and illicit drug consumption
}

Background: It is possible that men consume a higher amount of legal and illegal drugs than women. This can be assessed using the peer methodology, that is an adaptation of the privileged access interviewers (PAI) method and allows to identify populations in which there may be a tendency to under state a phenomenon (hidden populations) Aim: To compare the consumption of legal and illegal drugs by gender. Material and methods: Drug consumption was assessed using a peer methodology in university students between 18 and 26 years of age. Results: A random sample of 56 women (mean age 21.6 years) and 86 men (mean age 21.5 years), was studied. Women tended to report a higher proportion of tobacco consumption than men. Both genders had a similar consumption behavior of alcohol, total legal drugs, marihuana, cocaine, ecstasies and total illegal drugs. Among subjects that recognized the consumption of legal drugs, men have a higher proportion of illicit drug use and women have a higher proportion of smoking. Men have a higher awareness of the damages caused by drug consumption. Conclusions: The results in these small population sample do not support the hypothesis that men have a higher frequency and proportion of illicit drug consumption (Rev Méd Chile 2007; 135: 449-56).

(Key words: Alcohol drinking; Cannabis; Gender identity; Street drugs; Tobacco)

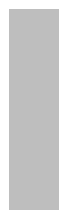

Recibido el 3 de enero, 2006. Aceptado el 28 de septiembre, 2006.

${ }^{1}$ Escuela de Salud Pública, Facultad de Medicina, Universidad de Chile. ${ }^{2}$ Escuela de Psicología, Universidad de Santiago de Chile.

aMaster en Estadística Matemática

bMagíster en Salud Pública

cDoctor en Psicología

L os problemas del adolescente son un motivo permanente de consultas a los profesionales del área de la salud mental en la actualidad. Las conductas de riesgo de estos jóvenes están asociadas al consumo de alcohol, marihuana, cocaína, éxtasis y otras sustancias químicas. Tales conductas tienen

Correspondencia a: Dr. Jorge Rodríguez T. Independencia 939. Santiago. Fax: 7774163. E mail: jrodrigu@med.uchile.cl, jrodrigu80@hotmail.com consecuencias muy complejas para la salud, en distintos tipos de morbilidad médica y psicosocial. Los factores causales pasan por lo individual, familiar, cultural y grupo de amigos ${ }^{1-5}$.

En el último peńodo, el uso y el abuso de drogas, así como el tipo de consumo, se ha convertido en un problema social que afecta la estabilidad de todas las instituciones que conforman una sociedad, en especial las sociales y políticas ${ }^{6-10}$.

En Chile, se han realizado un gran número de trabajos en este sentido, los que muestran la 
magnitud del problema, en especial el Consejo Nacional de Estupefaciente (CONACE), el cual realiza estudios a nivel nacional, regional y comunal, para evaluar la dimensión de este fenómeno en población general y escolar11-16.

El problema de drogadicción y sus consecuencias en el mundo y en particular en nuestro país, está lejos de ser algo resuelto, siendo esto avalado por todos los trabajos científicos desarmollados, tanto para drogas ilícitas como lícitas. Por ejemplo, en un estudio reciente llevado a cabo con adolescentes en Bangkok, se encontró que 5,4\% consume cigamillos, $37,3 \%$ consume alcohol y 37,8\% consume drogas ilícitas. La prevalencia del consumo de drogas en estudiantes universitarios de medicina, en países desarrollados, muestra una baja del consumo de cigarrillos (de $28,8 \%$ a $9,2 \%$ ), en contraste a una fuerte alza del consumo de alcohol. Asimismo, investigaciones recientes del consumo de drogas en universitarios de Sao Paulo, muestran que el consumo de drogas lícitas ha aumentado en mujeres, mientras que el consumo excesivo tiende a ser mayor en el sexo masculino (86,5\% para el tabaco y $65 \%$ para el alcohol). Por otro lado, se ha encontrado una fuerte relación explicativa, en un plazo de cinco años, entre el consumo de drogas, violencia y victimización. Dentro del consumo de drogas en nuestro país, el alcoholismo es una de las mayores preocupaciones, que está atacando cada vez con mayor fuerza a la población de jóvenes ${ }^{10,17-20}$.

En jóvenes de 19 a 25 años, los resultados no son alentadores. En 2004, los índices de consumo de drogas ilícitas eran similares a los de 2000 y superiores a 1994. El aumento del consumo de drogas ilícitas fue de 8,8\%, en relación al año 2000 y $61 \%$ a la obtenida en 1994 . Por otro lado, al igual que en la mayoría de los estudios, el consumo de drogas lícitas es muy superior al consumo de drogas ilícitas, independiente del género, situación también válida en los estudios de población escolar. Sin embargo, los valores obtenidos mantienen la incertidumbre acerca de si en la información hay una subdeclaración del consumo, en especial referente a drogas ilícitas, que llevase a subestimar la real magnitud del consumo de drogas ${ }^{11,16,21-23}$.

Existe una variedad de metodologías onientadas a ubicar poblaciones ocultas, como los consumidores de drogas, una de estas metodologías es la denominada entrevistador de acceso privilegiado (EAP), que ha sido utilizada con mucho éxito en Europa ${ }^{24-26}$. En nuestro país, se ha desarmollado una metodología similar, pero onientada específicamente a disminuir la subdeclaración de consumo de drogas ${ }^{23}$.

El objetivo de esta investigación es aportar más antecedentes relacionados con el comportamiento del consumo de drogas entre hombres y mujeres universitarios, pertenecientes a un nivel socioeconómico medio-alto y alto, aplicando una adaptación de la (EAP), que se denomina metodología de pares ${ }^{23,27}$. Esto, con el propósito de minimizar o eliminar la subdeclaración del consumo de drogas, mejorando la estimación de los índices de consumo. La hipótesis general que se plantea es que los hombres declararan consumir drogas en una mayor proporción y cantidad que el consumo declarado por las mujeres.

\section{MATERIAL Y MÉTODO}

La población a estudiar fueron jóvenes de 18 a 26 años, universitarios de nivel socioeconómico medio alto y alto (mensualidad mayor a $\$ 240.000$ pesos) que asistían a una universidad privada no tradicional de la Región Metropolitana, el año 2003.

Se seleccionaron dos universidades privadas no tradicionales, bajo el supuesto que la variabilidad del consumo al interior de éstas es mayor, que entre universidades. Estas dos instituciones accedieron a participar en el estudio, entregando información sobre número de alumnos por carrera y dando las facilidades de acceso para seleccionar tanto a los entrevistadores como a los entrevistados.

Se utilizó la metodología de pares en la recolección de los datos, que consiste en solicitar a las instituciones la realización del estudio y el contacto con alumnos voluntarios para la realización de la entrevista $^{23}$. Se presentaron 12 voluntarios, 6 de cada institución, quienes desconocían los objetivos del estudio. Se realizaron dos reuniones de capacitación con el grupo de voluntarios. En la primera sesión se explicó el objetivo del estudio, el instrumento a utilizar y la forma de aplicación; enfatizando el anonimato y confidencialidad que tiene la entrevista. En la segunda sesión se practicó la aplicación del instrumento, haciendo las veces de entrevistador y de entrevistado. Para minimizar posibles sesgos que pudieran afectar la subdeclaración o sobredeclaración del consumo de drogas, se asignó a los entrevistadores a facultades distintas a la que pertenecían, por lo tanto, el concepto de par 
se refiere a que los entrevistadores son alumnos de la misma institución.

Los entrevistadores fueron estudiantes de ambos géneros y con edades entre 18 y 22 años (el promedio de edad fue 21,5 con una desviación estándar de 1,9 años).

Se consideraron drogas lícitas el consumo de tabaco o alcohol. Se definió el consumo de tabaco, si éste se consumía al menos dos veces por semana; y para el alcohol o marihuana, que al menos se consumía una vez por semana ${ }^{27}$. Para el resto de las drogas (cocaína, éxtasis, pasta base u otra), se consideró consumo, la ingesta de, al menos, una vez al mes. Se consideró nivel socioeconómico medio-alto y alto a aquellos alumnos de universidades privadas con un arancel superior a \$240.000 mensuales.

La selección de la muestra fue considerando el género y se realizó a partir del listado de alumnos por facultad, en ambas universidades. El tamaño de la muestra se obtuvo a partir de un estudio de prevalencia sobre el consumo de drogas en estudiantes universitarios, considerando una tasa de consumo de $24,0 \%$, un nivel de confianza de 95,0\% y un error máximo de estimación de 7,0\%.

Debido a las restricciones de la metodología de pares, la entrevista se realizó en los patios de las universidades, donde se tiene acceso a todos los alumnos, independientemente de la carrera o nivel que cursen (se les indicó a los entrevistadores, para asegurar la aleatoriedad en la selección que entrevistara, uno de cada cinco hombres y una de cada cinco mujeres, hasta completar el tamaño muestral). En total, se entrevistaron 56 mujeres y 86 hombres de dos instituciones privadas, quienes accedieron voluntariamente a participar en el estudio, desistieron cinco hombres de las carreras de ingeniería civil, arquitectura y leyes y dos mujeres de las carreras de leyes y diseño, que representan 3,5\% de la muestra.

Los datos fueron ingresados al programa EPIINFO y en la descripción y análisis de datos se utilizó el paquete estadístico SPSS 12.0. Se obtuvieron indicadores descriptivos, intervalos de confianza de 95\% (bilateral y unilateral). Las pruebas estadísticas utilizadas obedecen básicamente a la condición de las variables y su distribución. Para estudiar asociación de variables cualitativas (consumo de drogas, sexo, conciencia del daño, conocer los efectos del consumo de drogas, policonsumidor) se utilizó la pruebas de Fisher y la prueba Z. En el caso de variables cuantitativas (edad, edad de inicio en el hábito, número de veces de consumo) se utilizaron las pruebas $t$ de Student y Wilcoxon. Las pruebas utilizadas fueron bilaterales y unilaterales, con nivel de significación máximo de $5 \%$.

\section{RESUlTADOS}

En la muestra total, 92,3\% de los jóvenes declaró consumir drogas lícitas (tabaco o alcohol), el consumo de tabaco alcanzó 78,9\% y el consumo de alcohol 80,3\%. En el caso de las drogas ilícitas, el consumo de marihuana fue de $63,4 \%$, cocaína $4,9 \%$ y éxtasis $2,1 \%$. Sólo 33,3\% de la muestra, declaró tener conciencia del daño que produce el consumo de drogas y $83,1 \%$ dijo conocer los efectos de las drogas.

El porcentaje de mujeres que consumía tabaco fue significativamente mayor que el porcentaje alcanzado por los hombres, siendo ésta la única droga lícita donde se encontró diferencias entre géneros, el consumo de alcohol en las mujeres (76,8\%), fue muy similar al de hombres (82,6\%), siendo la droga lícita de mayor consumo en los jóvenes (Tabla 1).

Todo joven que consumía alguna droga ilícita, independiente del género, también consumía manihuana, que sigue siendo la droga ilícita de mayor consumo, alcanzando 63,4\%. El consumo de hombres fue ligeramente mayor al de mujeres en las drogas ilícitas. Se observó además que los hombres tendían a consumir más drogas duras que las mujeres, de hecho, sólo una mujer consumía cocaína, mientras que hubo seis hombres que consumían cocaína y uno también consumía éxtasis (de los tres consumidores).

A nivel general, el consumo de drogas ilícitas y particularmente de marihuana, presentó un consumo de $58,9 \%$ de las mujeres y $66,3 \%$ en los hombres. El consumo de cocaína fue de 1,8\% para las mujeres y de 7,0\% en los hombres, y en el caso del éxtasis, sólo los hombres consumían este tipo de droga con 3,5\%. En drogas ilícitas no se detectaron diferencias significativas entre las tasas de consumo a nivel global y por tipo de droga. Los intervalos resultantes para el grupo total fueron un consumo de $55,5 \%$ a $71,3 \%$ para drogas ilícitas, $13,4 \%$ a $8,5 \%$ en cocaína y como máximo 4,1\% para éxtasis (Tabla 2).

$\mathrm{Al}$ considerar a los jóvenes consumidores que declaran consumir drogas lícitas, las mujeres a nivel general, consumían significativamente más tabaco que los hombres (94,2\% vs $79,7 \%)$. En cambio, los 
Tabla 1. D istribución del consumo de drogas lícitas según género

\begin{tabular}{|lrrrrrr|}
\hline Drogas lícitas & \multicolumn{2}{c}{ Femenino } & \multicolumn{2}{c}{ Masculino } & Total \\
& $\mathrm{n}$ & $\%$ & $\mathrm{n}$ & $\%$ & $\mathrm{n}$ & $\%$ \\
\hline Total lícitas* & 56 & 39,4 & 86 & 60,6 & 142 & 100,0 \\
$\quad$ no & 4 & 7,1 & 7 & 8,1 & 11 & 7,7 \\
$\quad$ sí & 52 & 92,9 & 79 & 91,9 & 131 & 92,3 \\
Fumar** & 7 & 12,5 & 23 & 26,7 & 30 & 21,1 \\
$\quad$ no & 49 & 87,5 & 63 & 73,3 & 112 & 78,9 \\
$\quad$ sí & & & & & & \\
Alcohol*** & 13 & 23,2 & 15 & 17,4 & 28 & 19,7 \\
$\quad$ no & 43 & 76,8 & 71 & 82,6 & 114 & 80,3 \\
$\quad$ sí & & & & & \\
\hline
\end{tabular}

*Fisher: $\mathrm{p}=0,5489$. $* \mathrm{Z}=2,02 \mathrm{p}=0,0217$. ** $\mathrm{Z}=0,84 \mathrm{p}=0,2005$.

Tabla 2. D istribución del consumo de drogas ilícitas según género

\begin{tabular}{|lrrrrrr|}
\hline \multirow{2}{*}{ Drogas } & \multicolumn{2}{c}{ Femenino } & \multicolumn{2}{c}{ Masculino } & \multicolumn{2}{c|}{ Total } \\
& $\mathrm{n}$ & $\%$ & $\mathrm{n}$ & $\%$ & $\mathrm{n}$ & $\%$ \\
\hline Total ilícitas* & 56 & 100,0 & 86 & 100,0 & 142 & 100,0 \\
$\quad$ no & 23 & 41,1 & 29 & 33,7 & 52 & 36,6 \\
sí & 33 & 58,9 & 57 & 66,3 & 90 & 63,4 \\
Cocaína** & & & & & & \\
$\quad$ no & 55 & 98,2 & 80 & 93,0 & 135 & 95,1 \\
sí & 1 & 1,8 & 6 & 7,0 & 7 & 4,9 \\
Exxtasis*** & & & & & & \\
$\quad$ no & 56 & 100,0 & 83 & 96,5 & 139 & 97,9 \\
sí & 0 & 0,0 & 3 & 3,5 & 3 & 2,1 \\
\hline
\end{tabular}

$* \mathrm{Z}=0,89 \mathrm{p}=0$,1867. *Fisher: $\mathrm{p}=0,1595$. **Fisher: $\mathrm{p}=0,2191$.

hombres presentaban mayor consumo de cocaína ( $0 \%$ vs $7,6 \%)$ y más conciencia del daño que produce este consumo. En los jóvenes que declararon no consumir drogas lícitas, las diferencias no alcanzaron niveles significativos (Tabla 3).

Dentro de los consumidores de tabaco, las mujeres se iniciaban en el hábito a una edad más temprana que los hombres, en cambio los hombres presentaban un mayor consumo de marihuana, ambas cifras significativas. Se observó asimismo, que los hombres tenían más conciencia que el consumo de tabaco es un problema para la salud y conocían mayor número de patologías asociadas al consumo. Por otro lado, en el grupo de los no fumadores no se observaron diferencias en los indicadores de consumo de drogas (Tabla 4).
En el grupo que declaró consumir alcohol, las mujeres comenzaron el consumo a una edad promedio más tardía que los hombres, y eran ellas las que tenían mayor prevalencia del hábito de fumar. Los hombres consumían alcohol un mayor número de veces que las mujeres y al igual que en el grupo de consumidores de tabaco tenían mayor conocimiento de las enfermedades asociadas a este consumo (Tabla 5).

En el grupo de consumidores de alguna droga ilícita no se observaron diferencias entre género, mientras que en el grupo que declaró no consumir drogas ilícitas, el porcentaje de mujeres con el hábito de fumar fue significativamente mayor que en los hombres, observándose la misma situación para los consumidores de marihuana (Tabla 6). 
Tabla 3. Características de los consumidores y no consumidores de drogas lícitas, según género

\begin{tabular}{|lrrrrc|}
\hline \multirow{2}{*}{ Droga lícita } & \multicolumn{2}{c}{ Femenino } & \multicolumn{2}{c|}{ Masculino } & \\
\hline Fumar & $\mathrm{n}$ & $\%$ & $\mathrm{n}$ & $\%$ & $\mathrm{p}$ \\
Poli consumidor & 49 & 94,2 & 63 & 79,7 & $0,0109^{*}$ \\
Alcohol (OH) & 40 & 76,9 & 55 & 69,6 & 0,1803 \\
D. Ilícita & 43 & 82,7 & 71 & 89,9 & 0,1116 \\
Marihuana (THC) & 30 & 57,7 & 56 & 70,9 & 0,0606 \\
Cocaína & 30 & 57,7 & 56 & 70,9 & 0,0606 \\
Éxtasis & 0 & & 6 & 7,6 & $0,0440^{*}$ \\
Conciencia del daño & 0 & & 3 & 3,8 & 0,2160 \\
Conocer los efectos & 12 & 23,1 & 32 & 40,5 & $0,0197 *$ \\
No consume drogas lícitas & 41 & 78,8 & 69 & 87,3 & 0,0882 \\
Droga ilícitas & & & & & \\
Marihuana (THC) & 3 & 75,0 & 1 & 14,3 & 0,0878 \\
Cocaína & 3 & 75,0 & 1 & 14,3 & 0,0878 \\
Conciencia del daño & 1 & 25,0 & 0 & & 0,3636 \\
Conocer los efectos & 2 & 50,0 & 2 & 28,6 & 0,4697 \\
\end{tabular}

*Z =pruebas unilaterales.

Tabla 4. Características de los consumidores y no consumidores de tabaco, según género

\begin{tabular}{|c|c|c|c|c|c|c|c|}
\hline \multirow[b]{2}{*}{ Fumador } & \multicolumn{3}{|c|}{ Femenino } & \multicolumn{3}{|c|}{ Masculino } & \multirow[b]{2}{*}{$\mathrm{p}$} \\
\hline & Pron & nedio & d.e. & Pro & hedio & d.e. & \\
\hline Edad inicio(años) + & $\begin{array}{r}15,1 \\
\text { № }\end{array}$ & $\%$ & 2,8 & $\begin{array}{r}15,9 \\
\mathrm{n}\end{array}$ & $\%$ & 2,6 & 0,0582* \\
\hline Marihuana (THC) & 30 & 61,2 & - & 48 & 76,2 & - & $0,0444^{* *}$ \\
\hline Cocaína & 0 & & - & 4 & 6,3 & - & 0,0959 \\
\hline Éxtasis & 0 & & - & 3 & 4,8 & - & 0,0871 \\
\hline Conciencia del daño & 12 & 24,5 & - & 26 & 41,3 & - & $0,0320^{* * *}$ \\
\hline Conocer los efectos & 38 & 77,6 & - & 57 & 90,5 & - & $0,0290^{* *}$ \\
\hline \multicolumn{8}{|l|}{ No fumador } \\
\hline Alcohol (OH) & 3 & 42,9 & - & 16 & 69,6 & - & 0,2004 \\
\hline D. ilícita & 3 & 42,9 & - & 9 & 39,1 & - & 0,5972 \\
\hline Marihuana (THC) & 3 & 42,9 & - & 9 & 39,1 & - & 0,5972 \\
\hline Cocaína & 1 & 14,3 & - & 2 & 8,7 & - & 0,5638 \\
\hline Conciencia del daño & 2 & 28,6 & - & 8 & 34,8 & - & 0,5711 \\
\hline Conocer los efectos & 7 & 100,0 & - & 16 & 69,6 & - & 0,1204 \\
\hline
\end{tabular}

+promedio; *t-student **; pruebas unilaterales.

Al comparar la conducta de los consumidores de cocaína según género, a pesar de las grandes diferencias que se observaron entre ambos grupos, éstas no alcanzaron a ser significativas al igual que en el grupo de consumidores de éxtasis, debido fundamentalmente a los pocos estudiantes que declararon consumir estos tipos de drogas. Por otro lado, en el grupo no consumidor de cocaína, el consumo de tabaco fue mayor en las mujeres y los hombres tenían mayor conciencia del daño, situación que también se observó en el grupo de no consumidores de éxtasis (Tabla 7). 
Tabla 5. C aracterísticas de los consumidores y no consumidores de alcohol, según género

\begin{tabular}{|c|c|c|c|c|c|c|c|}
\hline \multirow[b]{2}{*}{ Consumidor de alcohol (OH) } & \multicolumn{3}{|c|}{ Femenino } & \multicolumn{3}{|c|}{ Masculino } & \multirow[b]{2}{*}{$\mathrm{p}$} \\
\hline & Prol & nedio & d.e. & Prom & edio & d.e. & \\
\hline Edad inicio (años)+ & 15,9 & & 2,8 & 15,1 & & 2,1 & $0,0370^{\text {** }}$ \\
\hline \multirow[t]{2}{*}{ № de veces semanal+ } & 1,3 & & 0,9 & 1,8 & & 1,1 & $0,0115^{*}$ \\
\hline & $\mathrm{n}$ & $\%$ & & $\mathrm{n}$ & $\%$ & & \\
\hline Fumar & 40 & 93,0 & - & 55 & 77,5 & - & $0,0158^{* * 1 *}$ \\
\hline D. ilícita & 25 & 58,1 & - & 50 & 70,4 & - & 0,0918 \\
\hline Marihuana (THC) & 25 & 58,1 & - & 50 & 70,4 & - & 0,0918 \\
\hline Cocaína & 0 & & - & 5 & 7,0 & - & 0,0886 \\
\hline Éxtasis & 0 & & - & 1 & 1,4 & - & 0,6228 \\
\hline Conciencia del daño & 8 & 18,6 & - & 30 & 42,3 & - & $0,0005^{\text {*** }}$ \\
\hline Conocer los efectos & 35 & 81,4 & - & 62 & 87,3 & - & 0,1949 \\
\hline \multicolumn{8}{|l|}{ No consumidor alcohol $(\mathrm{OH})$} \\
\hline Fumar & 9 & 69,2 & - & 8 & 53,3 & - & 0,1944 \\
\hline D. ilícita & 8 & 61,5 & - & 7 & 46,7 & - & 0,2199 \\
\hline Marihuana (THC) & 8 & 61,5 & - & 7 & 46,7 & - & 0,2199 \\
\hline Cocaína & 1 & 7,7 & - & 1 & 6,7 & - & 0,7222 \\
\hline Éxtasis & 0 & & - & 2 & 13,3 & - & 0,2778 \\
\hline Conciencia del daño & 6 & 46,2 & - & 4 & 26,7 & - & 0,2491 \\
\hline Conocer los efectos & 10 & 76,9 & - & 11 & 73,3 & - & 0,5879 \\
\hline
\end{tabular}

+promedio; *t-student **wilcoxon ***; pruebas unilaterales.

Tabla 6. Indicadores de consumo de drogas ilícitas según género

\begin{tabular}{|lrrrrr|}
\hline & \multicolumn{2}{c}{$\begin{array}{c}\text { Femenino } \\
\text { Porcentaje }\end{array}$} & \multicolumn{2}{c|}{ Masculino } \\
& $\mathrm{n}$ & $\%$ & $\mathrm{n}$ & $\%$ & $\mathrm{p}$ \\
& & & & & \\
Droga Ilícita & 30 & 90,9 & 48 & 84,2 & 0,2873 \\
Fumador & 25 & 75,8 & 50 & 87,7 & 0,0708 \\
Alcohol (OH) & 25 & 75,8 & 42 & 73,7 & 0,4168 \\
Poli consumidor & 30 & 90,9 & 56 & 98,3 & 0,1377 \\
D. lícita & 1 & 3,0 & 5 & 8,8 & 0,2802 \\
Cocaína & 0 & & 3 & 5,3 & 0,2491 \\
Éxtasis & 12 & 36,4 & 30 & 52,6 & 0,0680 \\
Conciencia del daño & 30 & 90,9 & 52 & 91,2 & 0,6189 \\
Conocer los efectos & & & & & \\
No Droga Ilícita & 19 & 82,6 & 15 & 51,7 & $0,0100^{*}$ \\
Fumar & 18 & 78,3 & 21 & 72,4 & 0,3145 \\
Alcohol (OH) & 15 & 65,2 & 13 & 44,8 & 0,0708 \\
Poli consumidor & 22 & 95,7 & 23 & 79,3 & 0,0933 \\
D. lícita & 2 & 8,7 & 4 & 13,8 & 0,4527 \\
Conciencia del daño & 15 & 65,2 & 21 & 72,4 & \\
Conocer los efectos & & & &
\end{tabular}

*Normal (Z). 
Tabla 7. Indicadores de consumo de cocaína según género

\begin{tabular}{|c|c|c|c|c|c|}
\hline & \multicolumn{2}{|c|}{$\begin{array}{l}\text { Femenino } \\
\text { Porcentaje }\end{array}$} & \multicolumn{2}{|c|}{$\begin{array}{l}\text { Masculino } \\
\text { Porcentaje }\end{array}$} & $\mathrm{p}$ \\
\hline \multicolumn{6}{|l|}{ Cocaína } \\
\hline Fumar & 0 & & 4 & 66,7 & 0,4286 \\
\hline Alcohol (OH) & 0 & & 5 & 83,3 & 0,2857 \\
\hline D. lícita & 0 & & 6 & 100,0 & 0,1428 \\
\hline Marihuana (THC) & 1 & 100,0 & 5 & 83,3 & 0,2857 \\
\hline Extasis & 0 & & 1 & 16,7 & 0,2857 \\
\hline Conciencia del daño & 1 & 100,0 & 4 & 66,7 & 0,7143 \\
\hline \multicolumn{6}{|l|}{ No Cocaína } \\
\hline Fumar & 49 & 89,1 & 59 & 73,8 & $0,0145^{*}$ \\
\hline Alcohol (OH) & 43 & 78,2 & 66 & 82,5 & 0,2660 \\
\hline D. lícita & 52 & 94,6 & 73 & 91,3 & 0,3575 \\
\hline Conciencia del daño & 13 & 23,6 & 30 & 37,5 & $0,0446^{*}$ \\
\hline Conocer los efectos & 44 & 80,0 & 67 & 83,8 & 0,2880 \\
\hline
\end{tabular}

*Normal (Z).

\section{DisCUSIÓN}

Todos los estudios nacionales e internacionales muestran que el consumo de drogas está concentrado en los grupos de edades más jóvenes (18 a 25 años). Chile es coincidente con esto, sin embargo el consumo de drogas ilícitas se ubica en un rango ligeramente más amplio, que se extiende de los 14 a los 35 años. Esto justifica que la preocupación de los estudios de drogas esté enfocada principalmente a estos grupos, además, las investigaciones indican que el género masculino tiene indicadores de consumo superiores al femenino.

Los resultados de este estudio, a través de la metodología de pares, entregan indicadores de consumo de drogas superiores a lo observado por otras investigaciones, en especial aquellos datos obtenidos por CONACE. Independientemente del tipo de población, los índices obtenidos en este estudio, a nivel global, no avalan la afirmación de que el género masculino tiene los mayores índices de consumo. En la presente investigación, el hábito de fumar en el género femenino tiene mayores índices que el obtenido por los hombres, en contraposición con nuestra hipótesis. Sin embargo, los resultados son coincidentes con la estructura de consumo de drogas lícitas e ilícitas.

Por otro lado, el análisis del comportamiento frente al consumo de drogas de subpoblaciones de consumidores y de no consumidores, indican que los resultados de la subpoblación de fumadores coinciden con otros estudios, los cuales señalan que los hombres tienen mayores índices que las mujeres. En las otras subpoblaciones o no hay diferencias entre los géneros o no se detecta una tendencia en ningún sentido.

Las mujeres, en todas las subpoblaciones, presentan un mayor porcentaje frente al hábito de fumar que los hombres, como también se hace notar que los índices obtenidos en estas subpoblaciones son mayores a otros estudios realizados sobre el consumo de drogas por género. Esta tendencia nacional es coincidente con las tasas de consumo observadas en estudiantes universitarios de Sao Paulo, ya que en el mismo periodo de análisis que la presente investigación, también se encuentra un mayor consumo de tabaco en mujeres que en hombres ${ }^{19}$.

Una de las interrogantes fundamentales que emergen de los resultados de este trabajo, es determinar si los índices de consumo obtenidos para ambos géneros a través de esta metodología serían replicables en otras poblaciones.

De acuerdo a los resultados obtenidos sobre el consumo de drogas por género, se puede indicar que las diferencias del consumo tienden a anularse y por otro lado, los índices obtenidos a través de esta metodología son mayores a otros estudios. Todo lo anterior, plantea la posibilidad de que esta nueva 
metodología entregue indicadores que tienden a disminuir la subdeclaración del consumo. Esto sugiere profundizar la investigación utilizando esta metodología y ver la posibilidad de su implementa-

\section{REFERENCIAS}

1. Florenzano R. Familia y Salud de los jóvenes. Ediciones Universidad Católica de Chile. Santiago de Chile, 1995.

2. Florenzano R. El adolescente y sus conductas de riesgo. Ediciones Universidad Católica de Chile. Santiago de Chile, 1998.

3. Florenzano R, Vaidés M, Serrano T, Rodríguez J, RoizbiatT A. Desarrollo Yoico, Familia y Adolescencia. Rev de Psiquiatría y Salud Mental 2001; 34-40.

4. Nishimura T, Hishinuma E, Else I, Goebert E, Andrade N. Ethnicity and Adolescent Substance Use. Cultural Diversity and Ethnic Minority Psychology 2005; 11: 239-58.

5. Loas G, Gullbaud O, Perez-Díaz F, Verrier A, Stephan P, Lang F ET AL. Dependency and suicidality in addictive disorders. Psychiatry Research 2005; 137: 1103-11.

6. Oficina Panamericana de Salud, Noticias e información del Centenario. www.oas.org/defaultesp.htm

7. MC WhinteR, FLoREnZANo R. Comelatos psicosociales de la farmacología: Resultados de un estudio en Santiago de Chile. Rev Psiquiátrica 1998; 15: 10-22.

8. Ministerio de Salud, Encuesta Calidad de Vida. Gobierno de Chile. 2000.

9. Boletín de la Oficina Sanitaria Panamericana Drogas. 1989; 6.

10. Oea-CICAD (Comisión intemacional para el control del abuso de drogas). Estrategia en el Hemisferio. 2001.

11. Consejo Nacional para el Control de Estupefaciente (CONACE), Cuarto estudio nacional de drogas en población escolar de Chile, 2001.

12. Consejo Nacional para el Control de Estupefaciente (CONACE). Primer estudio nacional sobre drogas en población general de Chile. 1994.

13. Consejo Nacional para el Control de Estupefaciente (CONACE). Segundo estudio nacional sobre drogas en población general de Chile. 1996.

14. Consejo Nacional para el Control de Estupefaciente (CONACE). Tercer estudio nacional sobre drogas en población general de Chile. 1998.

15. Consejo Nacional para el Control de Estupefaciente (CONACE). Cuarto estudio nacional sobre drogas en población general de Chile. 2000. ción en los estudios donde sea evidente la subdeclaración, tanto para los estudios de prevalencia e incidencia como para la identificación de los individuos que tienen este tipo de características.

16. Consejo Nacional para el Control de Estupefaciente (CONACE). Quinto estudio nacional sobre drogas en población general de Chile. 2002.

17. Ruangkanchanasetr S, Plitponkarnpim A, Hetrakul P, KongSAKON R. Youth risk behavior survey: Bangkok, Thailand. J Adolescent Health 2005; 227-35.

18. Boland M, Fitzpatrick P, Scaldan E, Daly L, Herity B, HORGAN J ET AL. Trends in medical student use of tobacco, alcohol and drugs in an Irish university, 1973-2002. Drug and Alcohol Dependence 2002.

19. PiLon S, O’Brien B, Piedra K. The relationship between drugs use and risk behaviours in Brazilian university students. Rev Latino-am Enfermagem 2005; 1169-76.

20. Weiner M, Sussman S, Sun P, Dent C. Explaining the link between violence perpetration, victimization and drug use. Addictive Behaviors 2005; 1261-6.

21. Araneda JC, Cumsile F. Consumo de drogas en población escolar de Chile en alumnos de $8^{\underline{o}}$ básico a $4^{\circ}$ medio, 2003. Revista Chilena de Salud Pública 2004; 63-71.

22. Alvarado R, Neves L, Vidal A. Factores asociados al consumo de drogas entre estudiantes de enseñanza media en la región de Magallanes. Rev Chilena de Salud Pública 2003; 134-45.

23. Rodríguez J, Hernández E, Cumsile MA. Implementación de la metodología de pares para estimar el consumo de drogas lícitas e ilícitas. Rev Chilena de Salud Pública 2005; 20-4.

24. Griffiths P, Gossop M, Powis B, Strang J. Reaching hidden populations of drug users by privileged access interviewers: methodological and practical issues. Addiction 1993; 1617-26.

25. Kuebler D, Hausser D. The Swiss Hidden Population Study: practical and methodological aspects of data collection by privileged access interviewers. Addiction 1997; 325-34.

26. Fountain J, Griffiths P. Synthesis of qualitative research on drug use in European Union: report on an EMCDDA project. European Addiction Research 1999; 4-20.

27. RodrígueZ J, HerNÁNDEZ E. Análisis de la confiabilidad y validez de constructo de un instrumento que mide el consumo de drogas lícitas e ilícitas en estudiantes universitarios. Rev Terapia Psicológica 2005; 83-90. 